\title{
Synthesis of phenylacetaldehyde amidines and their intramolecular cyclization
}

\author{
Alessandro Contini, Emanuela Erba,* and Pasqualina Trimarco \\ Istituto di Chimica Organica "A. Marchesini” e Centro Interuniversitario di Ricerca sulle \\ Reazioni Pericicliche e Sintesi di Sistemi Etero e Carbociclici, Università degli Studi di Milano, \\ Via Venezian 21, 20133 Milano, Italy \\ E-mail: emanuela.erba@unimi.it
}

\begin{abstract}
The one-pot reaction of phenylacetaldehyde with a primary amine followed by tosyl azide yields the corresponding benzamidine $\mathbf{1}$ along with the $\mathrm{N}^{1}$-monosubstituted- $\mathrm{N}^{2}$-tosylformamidine 2 . The participation of $\alpha$-amino acid esters as reactants permitted the base-promoted intramolecular condensations of benzamidines $\mathbf{1 d}, \mathbf{1 e}, \mathbf{1 f}$ and $\mathbf{1 i}$ to give the corresponding 1,2-dihydropyrol-3ones 6a-6d, respectively. The combination of phenylacetaldehyde, a pair of heterocyclic secondary amines, and 4-nitrophenyl azide in turn led to the two dihydrotriazole derivatives, $\mathbf{4 b}$ and $\mathbf{4 c}$. Loss of nitrogen from $\mathbf{4}$ in refluxing toluene afforded trisubstituted benzamidines $\mathbf{5 b}$ and 5c, that also undergo base-promoted intramolecular condensation, forming 1,2-dihydropyrrol-3ones $7 \mathbf{a}$ and $7 \mathbf{b}$ in turn.
\end{abstract}

Keywords: Amidines, intramolecular cyclization, pyrrol-3-ones, multicomponent reactions

\section{Introduction}

Amidines are versatile starting materials in several synthetic schemes. Our group recently developed a simple and useful route to optically pure $\alpha$-amino acid amidines ${ }^{1}$ through a heterocyclic transformation. The cycloaddition of tosyl azide or aryl azides with enamines gives rise to 4,5-dihydrotriazole intermediates which convert into the expected branched amidines by nitrogen loss and substituent migration from the 5- to the 4 position. However, a different evolution for the 4,5-dihydrotriazole intermediates is also possible, indeed the corresponding linear amidines were obtained by alkyl diazomethane loss as represented in Scheme 1. 


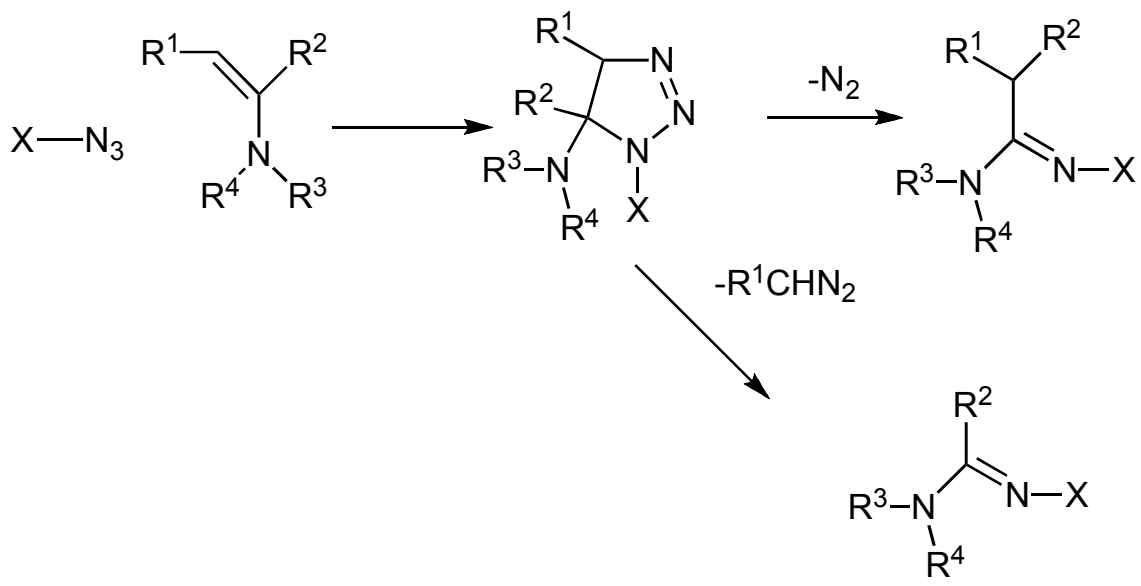

\section{Scheme 1}

Such a versatile multicomponent reaction has been extensively investigated by our group, ${ }^{2}$ but several aspects still remains unknown. For example, it is known that the nature of the starting carbonylic reactant appears to govern the outcome of the reaction. ${ }^{3}$ Aiming to thoroughly investigate the behaviour of phenylacetaldehyde as the carbonylic reactant, as well as to obtain material for new heterocyclic syntheses, several primary or secondary amines and tosyl- or 4nitrophenyl azide were reacted with phenylacetaldehyde according to Scheme 2.

\section{Results and Discussion}

The reaction of phenylacetaldehyde with tosyl azide and primary amines produced both $N$-tosyl2-benzylamidines 1a-i, with $\mathrm{N}_{2}$ loss, and $N$-tosyl-formamidines $\mathbf{2 a - i}$ with benzyldiazomethane loss. In all cases the 2-benzyl-amidine derivatives 1 were the main reaction products. It is already known that the reaction between phenylacetaldehyde and a secondary amine in presence of tosyl azide provides only formamidine. ${ }^{2 a}$ We verified the reaction trend by using proline methyl ester as the amine and the formamidine $\mathbf{3}$, bearing the proline moiety, was obtained as the only product. 

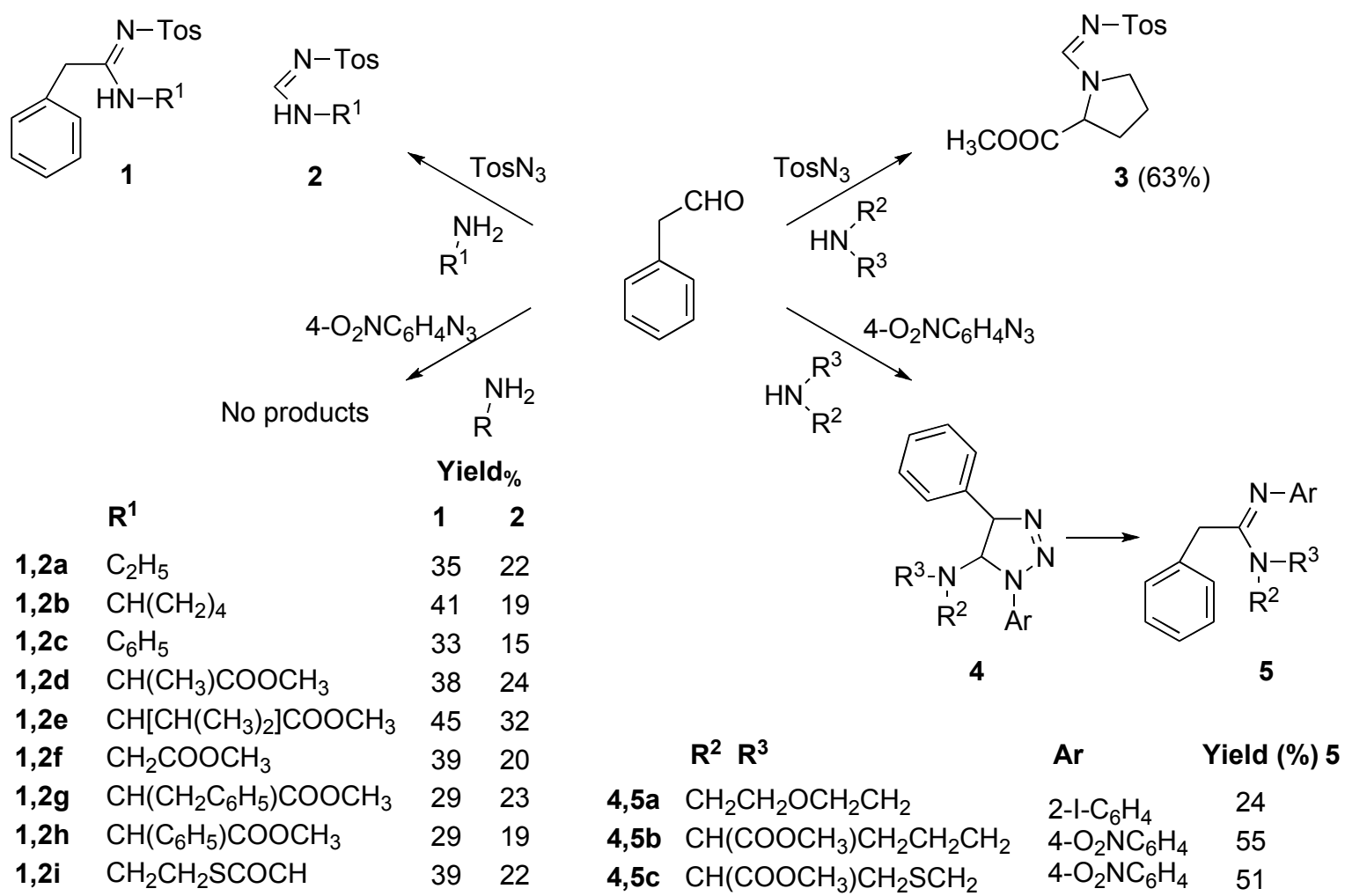

\section{Scheme 2}

The same conditions were chosen for the reaction with 4-nitrophenylazide, but an equimolar amount of phenylacetaldehyde, a suitable secondary amine and 4-nitrophenylazide yielded the stable triazoline 4 which, through prolonged heating, was transformed into the $N$-aryl-2benzylamidine derivatives $\mathbf{5}$ as the main products. No reaction occurred when phenylacetaldehyde was reacted with primary amines and 4-nitrophenylazide and the same behaviour was observed starting from $\alpha$-amino acid esters. As demonstrated below, this multicomponent reaction proved to be an efficient way to achieve useful intermediates for synthetic transformations. As the structures of the $N$-tosyl-benzylamidines $1 \mathbf{d}$-i, obtained from linear $\alpha$-amino acid esters, and of the aryl-benzylamidines $\mathbf{5 b}$-c, obtained from cyclic $\alpha$-amino acid esters, are quite similar, we proposed to take advantage of the $\alpha$-amidine methylene group, as well as of the presence of the ester moiety. By carrying out the reaction under basic conditions, an intramolecular condensation providing an heterocyclic ring could be expected between the nucleophilic methylene group ${ }^{4}$ and the ester function (Scheme 3).

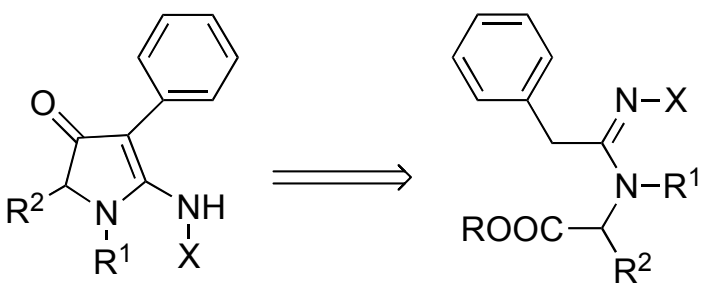

Scheme 3 
The prolonged heating of N-tosyl-benzylamidines 1a-d in a $1 \mathrm{M}$ solution of $t$-BuOK in THF supplied in good yield the 1H-2-methyl-4-phenyl-5-(4-methylbenzensulfonamide)-1,2dihydropyrrol-3-one $\mathbf{6 a}$ as a single product. The structure of $\mathbf{6 a}$ was supported by analytical and spectroscopic data. Besides the expected signals for the aromatic protons, the ${ }^{1} \mathrm{H}$ NMR spectrum showed at $4.10 \mathrm{ppm}$ a quartet associated with a proton coupled with a methyl group, confirmed by the ${ }^{13} \mathrm{C}$ signal at $57.1 \mathrm{ppm}$ associated with $\mathrm{CH}-2$. On the other hand the ${ }^{13} \mathrm{C} \mathrm{NMR}$ spectrum showed three quaternary carbons at 104.8, 166.1 and 180.4 ppm associated with C-4, C-5 and C3 , respectively. The analysis of the spectroscopic data infers the existence of the 3-oxo pyrrole tautomers, as recently reported by Friedrichsen et al. ${ }^{5}$ for similar structures. Likewise, amidines 1e,f,i heated in the same reaction conditions supplied the corresponding 1H-2-alkyl-4-phenyl-5(4-methylbenzensulfonamide)-1,2-dihydropyrrol-3-ones 6b-d (Scheme 4).

The $N$-aryl-benzylamidines $\mathbf{5 b}$,c also yielded the analogous bicyclic pyrrol-3-ones $\mathbf{7 a}, \mathbf{b}$ in basic medium. Unfortunately the basic reaction conditions gave rise to the loss of optical activity, explained by the enolization of the intermediate products.

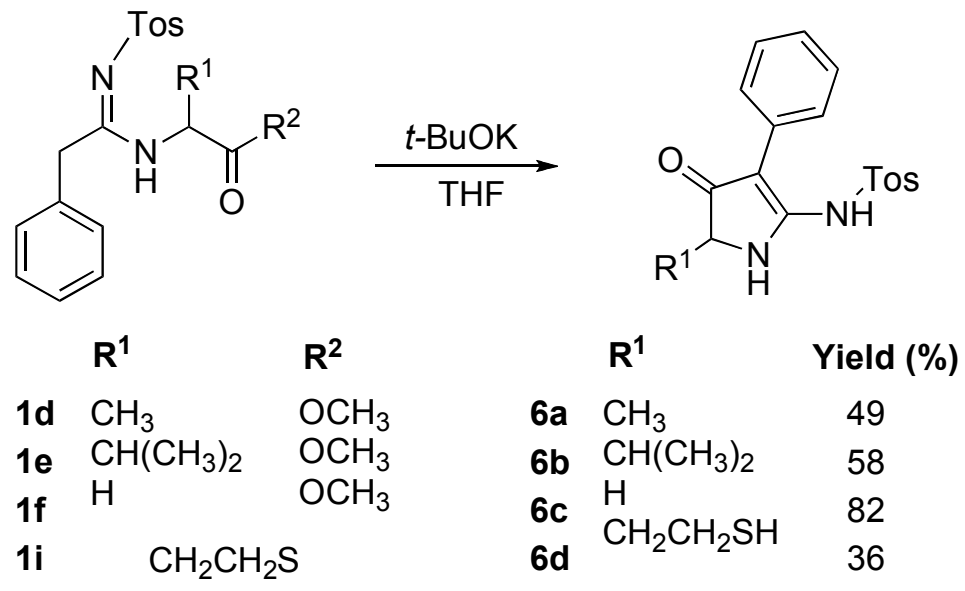<smiles>[X]CC1CCN1/C(Cc1ccccc1)=N\c1ccc([N+](=O)[O-])cc1</smiles>

$5 b, c$

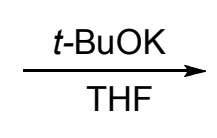

$\mathbf{R}^{1}$<smiles>[X]CC1C(=O)C(c2ccccc2)=C(Nc2ccc([N+](=O)[O-])cc2)N1C</smiles>

$7 a, b$

$\begin{array}{llr} & \mathbf{R}^{\mathbf{1}} & \text { Yield } \\ \mathbf{5 b}, \mathbf{7 a} & \mathrm{CH}_{2} & 45 \\ \mathbf{5 c}, \mathbf{7 b} & \mathrm{S} & 39\end{array}$

\section{Scheme 4}




\section{Conclusions}

In conclusion, in this work the chemical behaviour of phenylacetaldehyde was thoroughly investigated in multicomponent reactions with primary or secondary amines and various azides. The benzylamidines obtained proved to be useful intermediates in heterocyclic synthesis through a straightforward intramolecular condensation. Indeed starting from amidines $\mathbf{1 d - f , i}$ and $\mathbf{5 b}, \mathbf{c}$ a new pyrrol-3-ones series were synthesized.

\section{Experimental Section}

General Procedures. Mps were determined by a Büchi 510 (capillary) apparatus. IR spectra were measured with a JASCO IR Report 100 instrument (Nujol; $\mathrm{cm}^{-1}$ ). NMR spectra were obtained with Bruker Advance 300 and Varian Gemini 200 spectrometers in $\mathrm{CDCl}_{3}$ solution at $25{ }^{\circ} \mathrm{C}$, unless otherwise stated. $J$ values are given in $\mathrm{Hz}$. Low-resolution MS spectra were recorded with a Thermo-Finnigan LCQ ADVANTAGE AP electrospray/ion trap equipped instrument using a syringe pump device for the direct injection of sample solutions. 4-[3-(2iodophenyl)-5-phenyl-4,5-dihydro-3H-1,2,3-triazol-4-yl]morpholine $\mathbf{4 a ^ { 2 \mathrm { d } }}$ is a known compound.

\section{General procedure for the reaction of phenylacetaldehyde with tosylazide and primary or secondary amines}

The selected amine $(10 \mathrm{mmol})$ was dissolved in $20 \mathrm{ml}$ of $\mathrm{CH}_{2} \mathrm{Cl}_{2}$ and $7 \mathrm{~g}$ of $4 \AA$ molecular sieves were added to the solution. Phenylacetaldehyde $(10 \mathrm{mmol})$ and, after $30 \mathrm{~min}$., $10 \mathrm{mmol}$ of tosyl azide were added to the reaction mixture. The solution was stirred at room temperature for $12 \mathrm{~h}$ until disappearance of the starting materials (TLC: ethyl acetate/cyclohexane 1:1). The resulting suspension was filtered and evaporated. The crude product was purified by column chromatography (ethyl acetate/cyclohexane 1:1). When primary amines were used as reactants, two main fractions were collected containing 2-benzyl- $N$-alkyl- $N$-tosyl-amidine $\mathbf{1}$ and $N$-alkyl$N^{\prime}$-tosyl -formamidine $\mathbf{2}$, respectively. Starting from secondary amines, only formamidine $\mathbf{3}$ was obtained.

$\mathrm{N}$-Ethyl-2-phenyl-N'-tosylacetimidamide (1a). Yield 25\%. Yellow oil. ${ }^{1} \mathrm{H}$ NMR (200 MHz) 0.99 (t, J 7.3Hz, 3H, $\left.\mathrm{CH}_{3}\right), 2.39$ (s, $3 \mathrm{H}, \mathrm{CH}_{3} \mathrm{Ph}$ ), 3.20-3.33 (m, $\left.2 \mathrm{H}, \mathrm{CH}_{2}\right), 4.32$ (s, $2 \mathrm{H}, \mathrm{CH}_{2} \mathrm{Ph}$ ), $5.25(\mathrm{~s}, 1 \mathrm{H}, \mathrm{NH}), 7.09-7.87(\mathrm{~m}, 9 \mathrm{H}, \mathrm{ArH}) \mathrm{ppm} .{ }^{13} \mathrm{C} \mathrm{NMR}(50 \mathrm{MHz}) 13.7\left(\mathrm{CH}_{3}\right), 21.6\left(\mathrm{CH}_{3}\right)$, $37.2\left(\mathrm{CH}_{2}\right), 39.9\left(\mathrm{CH}_{2}\right), 126.5(\mathrm{CH}), 128.3(\mathrm{CH}), 129.4(\mathrm{CH}), 129.6(\mathrm{CH}), 130.3(\mathrm{CH}), 133.4$ (C), 141.1 (C), 142.3 (C), 166.7 (C) ppm. ESI-MS: $m / z 339$ [M+Na]. $\mathrm{C}_{17} \mathrm{H}_{20} \mathrm{~N}_{2} \mathrm{O}_{2} \mathrm{~S}$ (316.36): calcd. C 64.54, H 6.36, N 8.85\% found C 64.32, H 6.54, N 8.69\%

$N$-Ethyl- $N$ 'tosyl-formimidamide (2a). Yield $20 \%$. Mp $100-101^{\circ} \mathrm{C}$ (white crystals from $\mathrm{CH}_{2} \mathrm{Cl}_{2}$ and $\left.i-\mathrm{Pr}_{2} \mathrm{O}\right) .{ }^{1} \mathrm{H}$ NMR $(200 \mathrm{MHz}) 1.15-1.25\left(\mathrm{~m}, 3 \mathrm{H}, \mathrm{CH}_{3}\right), 2.40\left(\mathrm{~s}, 3 \mathrm{H}, \mathrm{CH}_{3} \mathrm{Ph}\right), 3.29-3.43(\mathrm{~m}$, $\left.2 \mathrm{H}, \mathrm{CH}_{2}\right), 6.80(\mathrm{sb}, 1 \mathrm{H}, \mathrm{NH}), 7.28$ e $7.56(2 \mathrm{~d}, 2+2 \mathrm{H}, 4 \mathrm{ArH}), 8.21(\mathrm{~d}, J=4.80 \mathrm{~Hz}, 1 \mathrm{H}, \mathrm{CH}) \mathrm{ppm}$. ${ }^{13} \mathrm{C}$ NMR (50 MHz) $13.9\left(\mathrm{CH}_{3}\right), 21.6\left(\mathrm{CH}_{3}\right), 36.9\left(\mathrm{CH}_{2}\right), 126.6(\mathrm{CH}), 129.8(\mathrm{CH}), 139.6(\mathrm{C})$, 
142.0 (C), $157.9(\mathrm{CH})$ ppm. ESI-MS: $m / z 249$ [M+Na], $225[\mathrm{M}-\mathrm{H}] . \mathrm{C}_{10} \mathrm{H}_{14} \mathrm{~N}_{2} \mathrm{O}_{2} \mathrm{~S}$ (226.24): calcd. C 53.09, H 6.24, N 12.38\% found C 52.93, H 6.50, N 12.21\%

$\mathrm{N}$-Cyclopentyl-2-phenyl- $\boldsymbol{N}^{\prime}$-tosylacetimidamide (1b). Yield $35 \%$. Mp $77-78{ }^{\circ} \mathrm{C}$. (white crystal from $\left.\mathrm{Et}_{2} \mathrm{O}\right) .{ }^{1} \mathrm{H}$ NMR $(200 \mathrm{MHz})$ 1.12-1.96 (m, 8H, 4CH $), 2.41\left(\mathrm{~s}, 3 \mathrm{H}, \mathrm{CH}_{3} \mathrm{Ph}\right), 4.09-4.19$ (m, $1 \mathrm{H}, \mathrm{CH}), 4.24\left(\mathrm{~s}, 2 \mathrm{H}, \mathrm{CH}_{2} \mathrm{Ph}\right), 5.15$ (bs, $\left.1 \mathrm{H}, \mathrm{NH}\right), 7.15-7.18$ (m 9H, ArH) ppm. ${ }^{13} \mathrm{C}$ NMR $(50$ MHz) $21.8\left(\mathrm{CH}_{3}\right), 23.9\left(\mathrm{CH}_{2}\right), 32.8\left(\mathrm{CH}_{2}\right), 34.1\left(\mathrm{CH}_{2}\right), 40.0\left(\mathrm{CH}_{2}\right), 53.8(\mathrm{CH}), 126.7(\mathrm{CH})$, $128.4(\mathrm{CH}), 129.5(\mathrm{CH}), 129.7(\mathrm{CH}), 130.3(\mathrm{CH}), 133.7(\mathrm{C}), 141.4(\mathrm{C}), 142.4(\mathrm{C}), 166.2(\mathrm{C})$ ppm. ESI-MS: $m / z 379$ [M+Na], 355 [M-H]. $\mathrm{C}_{20} \mathrm{H}_{24} \mathrm{~N}_{2} \mathrm{O}_{2} \mathrm{~S}$ (356.427): calcd. C 67.40, H 6.79, N $7.86 \%$ found $\mathrm{C} 67.28, \mathrm{H} 6.82$, N 7.71\%

$\boldsymbol{N}$-Cyclopentyl- $\boldsymbol{N}$ 'formimidamide (2b). Yield $18 \%$. Mp $116.117^{\circ} \mathrm{C}$ (white crystals from $\mathrm{Et}_{2} \mathrm{O}$ ). ${ }^{1} \mathrm{H}$ NMR (200 MHz) 1.25-2.02 (m, 8H, 4CH $)$, 2.39 (s, 3H, $\left.\mathrm{CH}_{3} \mathrm{Ph}\right), 4.16-4.29$ (m, 1H, CH), 6.47 (bs, 1H, NH), 7.22-7.81 (m, 4H, ArH), $8.24(\mathrm{~d} J=5.0 \mathrm{~Hz}, 1 \mathrm{H}, \mathrm{CH}) \mathrm{ppm} .{ }^{13} \mathrm{C}$ NMR $(50 \mathrm{MHz}) 21.6$ $\left(\mathrm{CH}_{3}\right), 23.6\left(\mathrm{CH}_{2}\right), 23.8\left(\mathrm{CH}_{2}\right), 32.7\left(\mathrm{CH}_{2}\right), 33.7\left(\mathrm{CH}_{2}\right), 53.6(\mathrm{CH}), 126.5(\mathrm{CH}), 129.5(\mathrm{CH})$, 139.8 (C), 142.6 (C), $157.6(\mathrm{CH})$ ppm. ESI-MS: $m / z 289[\mathrm{M}+\mathrm{Na}], 265[\mathrm{M}-\mathrm{H}] . \quad \mathrm{C}_{13} \mathrm{H}_{18} \mathrm{~N}_{2} \mathrm{O}_{2} \mathrm{~S}$ (266.301): calcd. C 58.63, H 6.81, N 10.52\% found C 58.50, H 6.99, N 10.39\%

$\boldsymbol{N}$-2-Diphenyl- $\boldsymbol{N}$-tosylacetimidamide (1c). Yield $24 \%$. Mp 161-162 ${ }^{\circ} \mathrm{C}$ (white crystal from $\mathrm{CH}_{2} \mathrm{Cl}_{2}$ and $i-\mathrm{Pr}_{2} \mathrm{O}$ ). ${ }^{1} \mathrm{H}$ NMR (200 MHz) $2.32\left(\mathrm{~s}, 3 \mathrm{H}, \mathrm{CH}_{3} \mathrm{Ph}\right), 4.26\left(\mathrm{~s}, 2 \mathrm{H}, \mathrm{CH}_{2} \mathrm{Ph}\right), 7.08-7.61$ (m, 10H, ArH), $10.46(\mathrm{~s}, 1 \mathrm{H}, \mathrm{NH}) \mathrm{ppm} .{ }^{13} \mathrm{C} \mathrm{NMR}(50 \mathrm{MHz}) 21.7\left(\mathrm{CH}_{3}\right), 40.5\left(\mathrm{CH}_{2}\right), 40.7\left(\mathrm{CH}_{2}\right)$, $121.7(\mathrm{CH}), 126.6(\mathrm{CH}), 127.3(\mathrm{CH}), 128.6(\mathrm{CH}), 129.0(\mathrm{CH}), 129.5(\mathrm{CH}), 129.8(\mathrm{CH}), 130.4$ $(\mathrm{CH}), 133.2(\mathrm{C}), 134.7(\mathrm{CH}), 136.9(\mathrm{CH}), 140.7(\mathrm{CH}), 163.7(\mathrm{CH}), 166.4(\mathrm{C}) \mathrm{ppm}$. ESI-MS: $\mathrm{m} / \mathrm{z}$ 387.4[M+Na], 363.6[M-H]. $\mathrm{C}_{21} \mathrm{H}_{20} \mathrm{~N}_{2} \mathrm{O}_{2} \mathrm{~S}$ (364.46): calcd. C 69.20, H 5.53, 7.69\% found $\mathrm{C}$ 69.12, H 5.77, N 7.48\%

$N$-Phenyl- $N$ 'tosyl-formimidamide (2c). Yield $12 \%$. Mp $197{ }^{\circ} \mathrm{C}$ (white crystal from $\mathrm{CH}_{2} \mathrm{Cl}_{2}$ and $\left.i \mathrm{Pr}_{2} \mathrm{O}\right) .{ }^{1} \mathrm{H}$ NMR $(200 \mathrm{MHz}) 2.41\left(\mathrm{~s}, 3 \mathrm{H}, \mathrm{CH}_{3} \mathrm{Ph}\right), 7.12-7.82(\mathrm{~m}, 10 \mathrm{H}, \mathrm{ArH}+\mathrm{NH}), 8.71(\mathrm{~d}$ $J=5.9 \mathrm{~Hz}, 1 \mathrm{H}, \mathrm{CH}) \mathrm{ppm} .{ }^{13} \mathrm{C}$ NMR $(50 \mathrm{MHz}) 21.6\left(\mathrm{CH}_{3}\right), 118.6(\mathrm{CH}), 121.4(\mathrm{CH}), 126.9(\mathrm{CH})$, $130.2(\mathrm{CH}), 138.8(\mathrm{C}), 139.8(\mathrm{C}), 143.0(\mathrm{C}), 155.2(\mathrm{CH}) \mathrm{ppm}$. ESI-MS: m/z $273[\mathrm{M}-\mathrm{H}]$. $\mathrm{C}_{14} \mathrm{H}_{14} \mathrm{~N}_{2} \mathrm{O}_{2} \mathrm{~S}$ (274.08): calcd. C, 61.29; H, 5.14; N, 10.21\% found C, 61.43; H, 5.21; N, $10.21 \%$

Methyl 2-(2-phenyl- $N$ '-tosylacetimidamido)propanoate (1d). Yield $28 \%$. Mp $95{ }^{\circ} \mathrm{C}$ (white crystal from $\left.\mathrm{Et}_{2} \mathrm{O}\right)$. [ $\left.\alpha\right]_{\mathrm{D}}=-6.3 .{ }^{1} \mathrm{H}$ NMR $(300 \mathrm{MHz}) 1.29\left(\mathrm{~d} J=7.3 \mathrm{~Hz}, 3 \mathrm{H}, \mathrm{CH}_{3}\right), 2.41(\mathrm{~s}, 3 \mathrm{H}$, $\mathrm{CH}_{3} \mathrm{Ph}$ ), 3.63 (s, $\left.3 \mathrm{H}, \mathrm{OCH}_{3}\right), 4.29$ (s, $\left.2 \mathrm{H}, \mathrm{CH}_{2} \mathrm{Ph}\right), 4.48-4.55(\mathrm{~m}, 1 \mathrm{H}, \mathrm{CH}), 5.87-5.90$ (bd, $1 \mathrm{H}$, $\mathrm{NH}), 7.21-7.84(\mathrm{~m}, 9 \mathrm{H}, \mathrm{ArH}) \mathrm{ppm} .{ }^{13} \mathrm{C} \mathrm{NMR}(75 \mathrm{MHz}) 17.5\left(\mathrm{CH}_{3}\right), 21.7\left(\mathrm{CH}_{3}\right), 39.8\left(\mathrm{CH}_{2}\right)$, $50.30\left(\mathrm{CH}_{3}\right), 52.76(\mathrm{CH}), 126.6(\mathrm{CH}), 128.4(\mathrm{CH}), 129.4(\mathrm{CH}), 129.6(\mathrm{CH}), 130.2(\mathrm{CH}), 133.0$ (C), 140.8 (C), 142.5 (C), 165.9 (C), 172.5 (C) ppm. ESI-MS: $m / z$ 373.3[M-H]. $\mathrm{C}_{19} \mathrm{H}_{22} \mathrm{~N}_{2} \mathrm{O}_{4} \mathrm{~S}$ (374.45): calcd. C 60.94, H 5.92, N 7.48\% found C 60.81, H 6.08, N 7.22\%

Methyl 2-( $\boldsymbol{N}^{\prime}$-tosylformimidamido)propanoate (2d). Yield $20 \%$. $\mathrm{Mp} 75^{\circ} \mathrm{C}$ (white crystals from $\mathrm{CH}_{2} \mathrm{Cl}_{2}$ and $\left.i-\mathrm{Pr}_{2} \mathrm{O}\right) .[\alpha]_{\mathrm{D}}=+9.25 .{ }^{1} \mathrm{H}$ NMR $(300 \mathrm{MHz}) 1.44\left(\mathrm{~d} J=7.3 \mathrm{~Hz}, 3 \mathrm{H}, \mathrm{CH}_{3}\right), 2.39(\mathrm{~s}, 3 \mathrm{H}$, $\left.\mathrm{CH}_{3} \mathrm{Ph}\right), 3.63\left(3 \mathrm{H}, \mathrm{s}, \mathrm{CH}_{3}\right), 4.61-4.68(\mathrm{~m}, 1 \mathrm{H}, \mathrm{CH}), 6.43-6.60$ (bs, $\left.1 \mathrm{H}, \mathrm{NH}\right), 7.24$ (d $J=8.4 \mathrm{~Hz}$, 2H, ArH), $7.74(\mathrm{~d} J=8.4,2 \mathrm{H}, \mathrm{ArH}), 8.27(\mathrm{~d} J=4.7,1 \mathrm{H}, \mathrm{CH}) \mathrm{ppm} .{ }^{13} \mathrm{C}$ NMR $(75 \mathrm{MHz}) .17 .7$ $\left(\mathrm{CH}_{3}\right), 21.6\left(\mathrm{CH}_{3}\right), 50.2\left(\mathrm{CH}_{3}\right), 52.9(\mathrm{CH}), 126.7(\mathrm{CH}), 129.5(\mathrm{CH}), 139.1(\mathrm{C}), 142.9(\mathrm{C}), 156.9$ 
(CH), 172.3 (C) ppm. ESI-MS: m/z 307 [M+Na], 283 [M-H]. $\mathrm{C}_{12} \mathrm{H}_{16} \mathrm{~N}_{2} \mathrm{O}_{4} \mathrm{~S}$ (248.33): calcd. C $50.69, \mathrm{H} 5.67, \mathrm{~N} 9.85 \%$ found C 50.84, H 5.60, N 9.68\%

Methyl 3-methyl-2-(2-phenyl- $N$ '-tosylacetamidamido)butanoate (1e). Yield 40\%. Mp $115{ }^{\circ} \mathrm{C}$ (yellow crystals from $\mathrm{CH}_{2} \mathrm{Cl}_{2}$ and $i$ - $\left.\mathrm{Pr}_{2} \mathrm{O}\right) .[\alpha]_{\mathrm{D}}=-2.8 .{ }^{1} \mathrm{H} \mathrm{NMR}(300 \mathrm{MHz}) 0.65(\mathrm{~d} J=6.9 \mathrm{~Hz}, 3 \mathrm{H}$, $\left.\mathrm{CH}_{3}\right), 0.74\left(\mathrm{~d} J=6.9 \mathrm{~Hz}, 3 \mathrm{H}, \mathrm{CH}_{3}\right), 2.00-2.21(\mathrm{~m}, 1 \mathrm{H}, \mathrm{CH}), 2.41\left(\mathrm{~s}, 3 \mathrm{H}, \mathrm{CH}_{3} \mathrm{Ph}\right), 3.61(\mathrm{~s}, 3 \mathrm{H}$, $\mathrm{CH}_{3}$ ), 4.32 (AB system $\left.J=17.6 \mathrm{~Hz}, 2 \mathrm{H}, \mathrm{CH}_{2} \mathrm{Ph}\right), 4.42-4.52(\mathrm{~m}, 1 \mathrm{H}, \mathrm{CH}), 5.66$ (bd, 1H, NH), 7.25-7.84 (m, 9H, ArH) ppm. ${ }^{13} \mathrm{C}$ NMR (75 MHz) $17.9\left(\mathrm{CH}_{3}\right), 18.9\left(\mathrm{CH}_{3}\right), 21.7(\mathrm{CH}), 30.9$ $\left(\mathrm{CH}_{3}\right), 39.9\left(\mathrm{CH}_{2}\right), 52.4(\mathrm{CH}), 126.5(\mathrm{CH}), 128.5(\mathrm{CH}), 129.4(\mathrm{CH}), 129.7(\mathrm{CH}), 130.3(\mathrm{CH})$, 133.0 (C), 140.7 (C), 142.5 (C), 166.6 (C), 171.3 (C) ppm. ESI-MS: m/s 425 [M+Na], 401[MH]. $\mathrm{C}_{21} \mathrm{H}_{26} \mathrm{~N}_{2} \mathrm{O}_{4} \mathrm{~S}$ (402.51): calcd. C 62.66, H 6.51, N 6.96\% found C 62.51, H 6.58, N 6.66\%

Methyl 3-methyl-2-(2-phenyl- $\boldsymbol{N}$-tosylformimidamido)butanoate (2e). Yield $30 \%$. Mp $95{ }^{\circ} \mathrm{C}$ (withe crystals from $\mathrm{CH}_{2} \mathrm{Cl}_{2}$ and $\left.i-\mathrm{Pr}_{2} \mathrm{O}\right) .[\alpha]_{\mathrm{D}}=-1.4 .{ }^{1} \mathrm{H} \mathrm{NMR}$ (300 MHz) $0.88-1.04(\mathrm{~m}, 6 \mathrm{H}$, $\left.2 \mathrm{CH}_{3}\right), 2.17-2.29(\mathrm{~m}, 1 \mathrm{H}, \mathrm{CH}), 2.39$ (s, 3H, $\left.\mathrm{CH}_{3} \mathrm{Ph}\right), 3.71\left(\mathrm{~s}, 3 \mathrm{H}, \mathrm{CH}_{3}\right), 4.67-4.70(\mathrm{~m}, 1 \mathrm{H}, \mathrm{CH})$, 6.35 (bs, 1H, NH), 7.48 (d, $J=8.0 \mathrm{~Hz}, 2 \mathrm{H}, \mathrm{ArH}), 7.73$ (d J=8.0Hz, 2H, ArH), 8.33 (d J=2.8Hz, $1 \mathrm{H}, \mathrm{CH})$ ppm. ${ }^{13} \mathrm{C}$ NMR $(75 \mathrm{MHz}) 18.0\left(\mathrm{CH}_{3}\right), 18.9\left(\mathrm{CH}_{3}\right), 21.7(\mathrm{CH}), 31.2\left(\mathrm{CH}_{3}\right), 52.6\left(\mathrm{CH}_{3}\right)$, $59.3(\mathrm{CH}), 126.7(\mathrm{CH}), 129.5(\mathrm{CH}), 139.1(\mathrm{C}), 142.9(\mathrm{C}), 157.9(\mathrm{CH}), 171.4(\mathrm{C}) \mathrm{ppm}$. ESI-MS: $\mathrm{m} / \mathrm{s} 335[\mathrm{M}+\mathrm{Na}], 311[\mathrm{M}-\mathrm{H}] . \mathrm{C}_{14} \mathrm{H}_{20} \mathrm{~N}_{2} \mathrm{O}_{4} \mathrm{~S}$ (312.38) calcd. C 53.83, H 6.45, N 8.97\% found C 53.70, H 6.64, N 8.72\%

Methyl 2-(2-phenyl- $\boldsymbol{N}^{\prime}$-tosylacetimidamido)acetate (1f). Yield 28\%. Yellow oil. ${ }^{1} \mathrm{H}$ NMR (200 $\mathrm{MHz}) 2.42\left(\mathrm{~s}, 3 \mathrm{H}, \mathrm{CH}_{3} \mathrm{Ph}\right), 3.63\left(\mathrm{~s}, 3 \mathrm{H}, \mathrm{CH}_{3}\right), 3.98$ (AB system, $\left.J=21.6 \mathrm{~Hz}, 2 \mathrm{H}, \mathrm{CH}_{2}\right), 4.32$ (s, $\left.2 \mathrm{H}, \mathrm{CH}_{2} \mathrm{Ph}\right), 5.85$ (bs, 1H, NH), 7.22-7.90 (m, 9H, ArH) ppm. ${ }^{13} \mathrm{C} \mathrm{NMR} \mathrm{(50} \mathrm{MHz)} 21.7\left(\mathrm{CH}_{3}\right)$, $39.7\left(\mathrm{CH}_{2}\right), 43.5\left(\mathrm{CH}_{2}\right), 52.7\left(\mathrm{CH}_{3}\right), 126.7(\mathrm{CH}), 128.4(\mathrm{CH}), 129.4(\mathrm{CH}), 129.7(\mathrm{CH}), 130.3$ (CH), 132.9 (C), 140.6 (C), 142.6 (C), 166.7 (C), 169.3 (C) ppm. ESI-MS: m/z 359 [M-H]. $\mathrm{C}_{18} \mathrm{H}_{20} \mathrm{~N}_{2} \mathrm{O}_{4} \mathrm{~S}$ (360.43): calcd. C 59.98, H 5.59, N 7.77\% found C 59.79, H 5.67, N 7.61\%

Methyl 2-( $\boldsymbol{N}$-tosylformimidamido)acetate (2f). Yield 22\%. Mp $85{ }^{\circ} \mathrm{C}$ (Yellow crystals from $\mathrm{CH}_{2} \mathrm{Cl}_{2}$ and $\left.i-\mathrm{Pr}_{2} \mathrm{O}\right) .{ }^{1} \mathrm{H} \mathrm{NMR}(200 \mathrm{MHz}) 2.40\left(\mathrm{~s}, 3 \mathrm{H}, \mathrm{CH}_{3} \mathrm{Ph}\right), 3.76\left(\mathrm{~s}, 3 \mathrm{H}, \mathrm{CH}_{3}\right), 4.13(\mathrm{~s}, 2 \mathrm{H}$, $\left.\mathrm{CH}_{2}\right), 6.44$ (bs, $\left.1 \mathrm{H}, \mathrm{NH}\right), 7.25$ and $7.75(2 \mathrm{~d} J=8.7 \mathrm{~Hz}, 2+2 \mathrm{H}, \mathrm{ArH}), 8.33(\mathrm{~d}, J=4.8 \mathrm{~Hz}, 1 \mathrm{H}, \mathrm{CH})$ ppm. ${ }^{13} \mathrm{C}$ NMR $(75 \mathrm{MHz}) 21.6\left(\mathrm{CH}_{3}\right), 43.2\left(\mathrm{CH}_{2}\right), 52.9\left(\mathrm{CH}_{3}\right), 126.9(\mathrm{CH}), 129.6(\mathrm{CH}), 138.9$ (C), 143.1 (C), $157.3(\mathrm{CH}), 169.2$ (C) ppm. ESI-MS: $m / z 293[\mathrm{M}+\mathrm{Na}] . \mathrm{C}_{11} \mathrm{H}_{14} \mathrm{~N}_{2} \mathrm{O}_{4} \mathrm{~S}(270.3)$ : calcd C 48.88, H 5.22; N 10.36\% found C 48.81, H 5.37, N 10.21\%

Methyl 3-phenyl-2-(2-phenyl- $N$-tosylacetimido)propanoate (1g). Yield 27\%. Mp $138{ }^{\circ} \mathrm{C}$ (whitish crystals from $\left.\mathrm{Et}_{2} \mathrm{O}\right) .[\alpha]_{\mathrm{D}}=+42.4{ }^{1} \mathrm{H}$ NMR $(200 \mathrm{MHz}) 2.43\left(\mathrm{~s}, 3 \mathrm{H}, \mathrm{CH}_{3} \mathrm{Ph}\right), 2.97(\mathrm{ABX}$ system $J=5.5,5.9$ and $\left.13.9 \mathrm{~Hz}, 2 \mathrm{H}, \mathrm{CH}_{2} \mathrm{Ph}\right), 3.61\left(\mathrm{~s}, 3 \mathrm{H}, \mathrm{CH}_{3}\right), 4.34(\mathrm{AB}$ system $J=17.59 \mathrm{~Hz}, 2 \mathrm{H}$, $\mathrm{CH}_{2}$ ), 4.72 (dd $J=5.5$ and $\left.5.9 \mathrm{~Hz}, 1 \mathrm{H}, \mathrm{CH}\right), 5.68(\mathrm{bs}, 1 \mathrm{H}, \mathrm{NH}), 6.72-7.85(\mathrm{~m}, 14 \mathrm{H}, \mathrm{ArH}) \mathrm{ppm}$. ${ }^{13} \mathrm{C}$ NMR $(50 \mathrm{MHz}) 21.7\left(\mathrm{CH}_{3}\right), 36.8\left(\mathrm{CH}_{2}\right), 39.8\left(\mathrm{CH}_{2}\right), 52.6\left(\mathrm{CH}_{3}\right), 55.2(\mathrm{CH}), 126.6(\mathrm{CH})$, $127.4(\mathrm{CH}), 128.4(\mathrm{CH}), 128.9(\mathrm{CH}), 129.1(\mathrm{CH}), 129.4(\mathrm{CH}), 129.6(\mathrm{CH}), 130.2(\mathrm{CH}), 132.7$ (C), 135.1 (C), 140.8 (C), 142.5 (C), 165.9 (C), 171.0 (C) ppm. ESI-MS: m/z 473 [M+Na], 449 [M-H]. $\mathrm{C}_{22} \mathrm{H}_{26} \mathrm{~N}_{2} \mathrm{O}_{4} \mathrm{~S}$ (450.55): calcd. C 66.64, H 5.82, N 6.22\% found $\mathrm{C} 66.58, \mathrm{H} \mathrm{5.98,} \mathrm{N}$ $6.07 \%$ 
Methyl 3-phenyl-2-( $\boldsymbol{N}^{\prime}$-tosylformimidamido)propanoate (2g). Yield 25\%. Mp $110{ }^{\circ} \mathrm{C}$ (white crystals from $\mathrm{CH}_{2} \mathrm{Cl}_{2}$ and $\mathrm{Et}_{2} \mathrm{O}$ ). $[\alpha]_{\mathrm{D}}=+155.1 .{ }^{1} \mathrm{H} \mathrm{NMR}$ (200 MHz). 2.41 (s, 3H, $\left.\mathrm{CH}_{3}\right), 3.19$ $\left(\mathrm{ABX}\right.$ system $J=5.1,5.5$ and $\left.14.3 \mathrm{~Hz}, 2 \mathrm{H}, \mathrm{CH}_{2} \mathrm{Ph}\right), 3.73\left(\mathrm{~s}, 3 \mathrm{H}, \mathrm{CH}_{3}\right), 4.93(\mathrm{dd}, J=5.1$ and $5.5 \mathrm{~Hz}$, $1 \mathrm{H}, \mathrm{CH}), 6.22(\mathrm{bs}, 1 \mathrm{H}, \mathrm{NH}), 6.92-7.82(\mathrm{~m}, 9 \mathrm{H}, \mathrm{ArH}), 8.26(\mathrm{~d} J=3.6 \mathrm{~Hz}, 1 \mathrm{H}, \mathrm{CH}) \mathrm{ppm} .{ }^{13} \mathrm{C}$ NMR $(50 \mathrm{MHz}) 21.7\left(\mathrm{CH}_{3}\right), 37.0\left(\mathrm{CH}_{2}\right), 52.8\left(\mathrm{CH}_{3}\right), 55.2(\mathrm{CH}), 126.8(\mathrm{CH}), 127.5(\mathrm{CH}), 128.8(\mathrm{CH})$, $129.4(\mathrm{CH}), 129.6(\mathrm{CH}), 135.1(\mathrm{C}), 139.1(\mathrm{C}), 143.0(\mathrm{C}), 156.8(\mathrm{CH}), 170.9(\mathrm{C})$ ppm. ESI-MS: m/s 383 [M+Na], 359 [M-H]. $\mathrm{C}_{18} \mathrm{H}_{20} \mathrm{~N}_{2} \mathrm{O}_{4} \mathrm{~S}$ (360.43): calcd. C 59.98, H 5.59, H 7.77\% found C 59.74, H 5.66, N 7.54\%

Methyl 2-phenyl-2-(2-phenyl- $N$ '-tosylacetimidamido)acetate (1h). Yield 20\%. Mp $60{ }^{\circ} \mathrm{C}$ (yellow crystals from $\left.\mathrm{Et}_{2} \mathrm{O}\right) .[\alpha]_{\mathrm{D}}=-57.3 .{ }^{1} \mathrm{H} \mathrm{NMR}(200 \mathrm{MHz}) 2.41\left(\mathrm{~s}, 3 \mathrm{H}, \mathrm{CH}_{3} \mathrm{Ph}\right), 3.58(\mathrm{~s}, 2 \mathrm{H}$, $\mathrm{CH}_{2}$ ), 4.31 (AB system $J=17.6 \mathrm{~Hz}, 2 \mathrm{H}, \mathrm{CH}_{2}$ ), 5.40 (d, $\left.J 6.3 \mathrm{~Hz}, 1 \mathrm{H}, \mathrm{CH}\right), 6.33$ (bd $J=6.3,1 \mathrm{H}$, $\mathrm{CH}), 7.05-7.63(\mathrm{~m}, 14 \mathrm{H}, \mathrm{ArH}) \mathrm{ppm} .{ }^{13} \mathrm{C} \mathrm{NMR}(50 \mathrm{MHz}) 21.7\left(\mathrm{CH}_{3}\right), 39.8\left(\mathrm{CH}_{2}\right), 53.1\left(\mathrm{CH}_{3}\right)$, $58.5(\mathrm{CH}), 126.5(\mathrm{CH}), 127.4(\mathrm{CH}), 128.5(\mathrm{CH}), 128.9(\mathrm{CH}), 129.1(\mathrm{CH}), 129.2129 .7(\mathrm{CH})$, $130.2(\mathrm{CH}), 133.0(\mathrm{C}), 135.5(\mathrm{C}), 140.6(\mathrm{C}), 142.4$ (C), $165.5(\mathrm{C}), 170.5$ (C) ppm. ESI-MS: $\mathrm{m} / z$ $459[\mathrm{M}+\mathrm{Na}], 435[\mathrm{M}-\mathrm{H}] . \quad \mathrm{C}_{24} \mathrm{H}_{24} \mathrm{~N}_{2} \mathrm{O}_{4} \mathrm{~S}$ (436.52): calcd. C 66.03, H 5.54, N 6.42\% found $\mathrm{C}$ 65.98, H 5.66, N 6.32\%

Methyl 2-phenyl-2-( $\boldsymbol{N}^{\prime}$-tosylformimidate)acetate (2h). Yield $18 \%$. Mp $104{ }^{\circ} \mathrm{C}$ (whitish crystals from $\mathrm{CH}_{2} \mathrm{Cl}_{2}$ and $\left.i-\mathrm{Pr}_{2} \mathrm{O}\right)$. $[\alpha]_{\mathrm{D}}=-86.92 .{ }^{1} \mathrm{H} \mathrm{NMR}(200 \mathrm{MHz}) 2.34\left(\mathrm{~s}, 3 \mathrm{H}, \mathrm{CH}_{3}\right), 3.69$ (s, $\left.3 \mathrm{H}, \mathrm{CH}_{3}\right), 5.62(\mathrm{~d} J=7.0 \mathrm{~Hz}, 1 \mathrm{H}, \mathrm{CH}), 6.91(\mathrm{bs}, 1 \mathrm{H}, \mathrm{NH}), 7.15-7.82(\mathrm{~m}, 9 \mathrm{H}, \mathrm{ArH}), 8.29(\mathrm{~d} J=4.7$ $\mathrm{Hz}, 1 \mathrm{H}, \mathrm{CH}) \mathrm{ppm} .{ }^{13} \mathrm{C}$ NMR $(50 \mathrm{MHz}) 21.7\left(\mathrm{CH}_{3}\right), 53.3\left(\mathrm{CH}_{3}\right), 58.3(\mathrm{CH}) ; 126.6(\mathrm{CH}), 127.7$ $(\mathrm{CH}), 129.1(\mathrm{CH}), 129.4(\mathrm{CH}), 135.1(\mathrm{C}), 139.1(\mathrm{C}), 140.8(\mathrm{C}), 156.6(\mathrm{CH}), 170.4(\mathrm{C}) \mathrm{ppm}$.

ESI-MS: $m / z 345$ [M-H]. $\quad \mathrm{C}_{17} \mathrm{H}_{18} \mathrm{~N}_{2} \mathrm{O}_{4} \mathrm{~S}$ (346.40): calcd. C 58.94, H 5.24, N 8.09\% found C $58.77, \mathrm{H} 5.46, \mathrm{~N} 7.98 \%$

$\boldsymbol{N}$-(2-Oxo-tetrahydrothiophen-3-yl)-2-phenyl- $\boldsymbol{N}$-tosylacetimidamide (1i). Yield 35\%. Mp $135{ }^{\circ} \mathrm{C}$ (light pink crystals from $\mathrm{CH}_{2} \mathrm{Cl}_{2}$ and $\left.i \mathrm{Pr}_{2} \mathrm{O}\right) .{ }^{1} \mathrm{H} \mathrm{NMR}(200 \mathrm{MHz}) 1.65-1.87(\mathrm{~m}, 1 \mathrm{H}$, $\mathrm{CH}), 3.41\left(\mathrm{~s}, 3 \mathrm{H}, \mathrm{CH}_{3} \mathrm{Ph}\right), 2.82-2.94(\mathrm{~m}, 1 \mathrm{H}, \mathrm{CH}), 3.11-3.48\left(\mathrm{~m}, 2 \mathrm{H}, \mathrm{CH}_{2}\right), 4.26$ (AB system $\left.J=17.6 \mathrm{~Hz}, 2 \mathrm{H}, \mathrm{CH}_{2} \mathrm{Ph}\right), 4.36-4.52(\mathrm{~m}, 1 \mathrm{H}, \mathrm{CHN}), 5.74$ (bs, 1H, NH), 7.12-7.82 (m, 9H, ArH) ppm. ${ }^{13} \mathrm{C}$ NMR (50 MHz) $21.7\left(\mathrm{CH}_{3}\right), 27.7\left(\mathrm{CH}_{2}\right), 31.1\left(\mathrm{CH}_{2}\right), 39.6\left(\mathrm{CH}_{2}\right), 61.1(\mathrm{CH}), 126.7$ $(\mathrm{CH}), 128.5(\mathrm{CH}), 129.6(\mathrm{CH}), 129.7(\mathrm{CH}), 130.2(\mathrm{CH}), 132.6(\mathrm{C}), 140.3(\mathrm{C}), 142.8(\mathrm{C}), 167.3$ (C), 203.9 (C) ppm. ESI-MS: $m / z 388$ [M-H]. $\mathrm{C}_{19} \mathrm{H}_{20} \mathrm{~N}_{2} \mathrm{O}_{3} \mathrm{~S}_{2}$ (388.5): calcd. C 58.74, H 5.19, N $7.21 \%$ found C 58.80, H 5.14, N 7.50\%

$\boldsymbol{N}$-(2-Oxo-tetrahydrothiophen-3-yl)- $\boldsymbol{N}$ '-tosylformimidamide (2i). Yield $15 \%$. Mp $143{ }^{\circ} \mathrm{C}$ (white crystals from $\mathrm{CH}_{2} \mathrm{Cl}_{2}$ and $i$ - $\mathrm{Pr}_{2} \mathrm{O}$ ). ${ }^{1} \mathrm{H} \mathrm{NMR}(200 \mathrm{MHz})$ 1.92-2.08 (m, 1H, CH), 2.39 (s, $\left.3 \mathrm{H}, \mathrm{CH}_{3} \mathrm{Ph}\right), 2.86-3.17(\mathrm{~m}, 1 \mathrm{H}, \mathrm{CH}), 3.21-3.41\left(\mathrm{~m}, 2 \mathrm{H}, \mathrm{CH}_{2}\right), 4.41-4.66(\mathrm{~m}, 1 \mathrm{H}, \mathrm{CHN}), 6.85$ (bs, 1H, NH), 7.04-7.74 (m, 4H, ArH), 8.35 (d, $J=4.7 \mathrm{~Hz}, 1 \mathrm{H}, \mathrm{CH}) \mathrm{ppm} .{ }^{13} \mathrm{C}$ NMR (50 MHz) 21.7 $\left(\mathrm{CH}_{3}\right), 27.8\left(\mathrm{CH}_{2}\right), 31.1\left(\mathrm{CH}_{2}\right), 60.9(\mathrm{CH}), 126.8(\mathrm{CH}), 129.7(\mathrm{CH}), 138.6(\mathrm{C}), 134.3(\mathrm{C}), 158.1$ $(\mathrm{CH}), 208.9$ (C) ppm. ESI-MS: $m / z 299[\mathrm{M}+\mathrm{H}] . \mathrm{C}_{12} \mathrm{H}_{14} \mathrm{~N}_{2} \mathrm{O}_{3} \mathrm{~S}_{2}$ (298.38): calcd. C 48.30, H 4.73, N 9.39\% found C 48.19, H 4.89, N 9.17\%

Methyl 1-[(tosylimino)methyl]-pyrrolidine-2-carboxylate (3). Yield 62\%. Mp 124-125 ${ }^{\circ} \mathrm{C}$ (white crystals from $\left.i-\mathrm{Pr}_{2} \mathrm{O}\right) .{ }^{1} \mathrm{H}$ NMR $(200 \mathrm{MHz}) 1.85-2.42\left(\mathrm{~m}, 6 \mathrm{H}, 3 \mathrm{CH}_{2}\right), 3.52-3.62(\mathrm{~m}, 3 \mathrm{H}$, 
CHPh), 3.78-3.82 (s, 3H, $\left.\mathrm{CH}_{3}\right), 4.39-4.49$ (m, 1H, CH), 7.31 e 7.78 (2d, J=8. Hz, 4H, ArH), 8.39 $(\mathrm{s}, 1 \mathrm{H}, \mathrm{CH}) \mathrm{ppm} .{ }^{13} \mathrm{C}$ NMR $(75 \mathrm{MHz})(E$ and $Z$ isomers $) 21\left(\mathrm{CH}_{3}\right), 23.2$ and $24.2\left(\mathrm{CH}_{2}\right), 29.8$ and $29.9\left(\mathrm{CH}_{2}\right), 47.3$ and $50.4\left(\mathrm{CH}_{2}\right), 52.5$ and $53.6(\mathrm{CH}), 59.4$ and $52.0\left(\mathrm{CH}_{3}\right), 126.6$ and 126.8 $(\mathrm{CH}), 129.4$ and $129.6(\mathrm{CH}), 139.5$ and $139.6(\mathrm{C}), 142.6$ and $142.7(\mathrm{CH}), 156.5$ and $157.7(\mathrm{C})$, 171.2 and 171.4 (C) ppm. $\mathrm{C}_{14} \mathrm{H}_{18} \mathrm{~N}_{2} \mathrm{O}_{4} \mathrm{~S}$ (310.37): calcd. C 54.18, H 5.85, N 9.03\% found C $54.37, \mathrm{H} 5.88, \mathrm{~N} 8.88 \%$

General procedure for the reaction of phenylacetaldehyde with 4-nitrophenyl azide and secondary amines

The selected amine $\left(6 \mathrm{mmol}\right.$ ) was dissolved in $20 \mathrm{~mL}$ of $\mathrm{CH}_{2} \mathrm{Cl}_{2}$ and $7 \mathrm{~g}$ of $4 \AA$ molecular sieves were added (if the amine used was a hydrochloride, $10 \mathrm{mmol}$ of TEA were added). Then, to the mixture, phenylacetaldehyde $(6 \mathrm{mmol})$ was added and, after $30 \mathrm{~min}$, 4-nitrophenylazide $(6 \mathrm{mmol})$. The mixture was stirred for $20 \mathrm{~h}$ until disappearance of the starting materials (TLC ethyl acetate/ cycloexane 1:1). The reaction mixture was filtered, washed with fresh water, dried with $\mathrm{Na}_{2} \mathrm{SO}_{4}$ and evaporated at reduced pressure. The residue was purified by chromatography over silica gel to supply triazoline 4.

Methyl 1-[(4S,5R)3-(4-nitrophenyl)-5-phenyl-4,5-dihydro-3H-1,2,3,triazol-4-yl]pyrrolidine2-carboxylate and methyl 1-[(4R,5S)3-(4-nitrophenyl)-5-phenyl-4,5-dihydro-3H-1,2,3,triazol-4-yl] pyrrolidine-2-carboxylate (4b). Yield 48\%, Mp $135{ }^{\circ} \mathrm{C}$ (white crystals from $i$ - $\operatorname{Pr}_{2} \mathrm{O}$ ). ${ }^{1} \mathrm{H}$ NMR 1.75-2.78 (m, $\left.6 \mathrm{H}, 3 \mathrm{CH}_{2}\right), 3.57-3.69(\mathrm{~m}, 1 \mathrm{H}, \mathrm{CH}), 3.57$ and $3.77\left(2 \mathrm{~s}, 3 \mathrm{H}, \mathrm{CH}_{3}\right), 5.18$ and $5.19(2 \mathrm{~d}$ $J=3.1$ and $J=3.3 \mathrm{~Hz}, 1 \mathrm{H}, \mathrm{CHPh}), 5.49$ and $5.68(2 \mathrm{~d} J=3.3$ and $J=3.1 \mathrm{~Hz}, 1 \mathrm{H}, \mathrm{CHN}), 7.00-8.34$ (m, 9H, ArH) ppm. ${ }^{13} \mathrm{C}$ NMR $23.4\left(\mathrm{CH}_{2}\right), 30.2\left(\mathrm{CH}_{2}\right), 45.8\left(\mathrm{CH}_{2}\right), 52.4(\mathrm{CH}), 59.4\left(\mathrm{CH}_{3}\right), 76.0$ $(\mathrm{CH}), 81.1(\mathrm{CH}), 115.5(\mathrm{CH}), 125.7(\mathrm{CH}), 126.8(\mathrm{CH}), 128.8(\mathrm{CH}), 129.6(\mathrm{CH}), 136.4(\mathrm{C}), 142.9$ (C), 144.9 (C), 173.5 (C) ppm. $\mathrm{C}_{20} \mathrm{H}_{21} \mathrm{~N}_{5} \mathrm{O}_{4}$ (395.41): calcd. $\mathrm{C}$ 60.75, $\mathrm{H} \mathrm{5.35,} \mathrm{N} \mathrm{17.71 \%} \mathrm{found}$ C $60.54, \mathrm{H} 5.55, \mathrm{~N} 17.66 \%$

Methyl 3-[(4S,5R)3-(4-nitrophenyl)5-phenyl-4,5-dihydro-3H-1,2,3-triazol-4-yl]-thiazolidine-4carboxylate and methyl 3-[(4R,5S)3-(4-nitrophenyl)5-phenyl-4,5-dihydro-3H-1,2,3-triazol-4-yl]thiazolidine-4-carboxylate (4c). Yield 47\%. Mp $95{ }^{\circ} \mathrm{C}$ (white crystals from $i$ - $\mathrm{Pr}_{2} \mathrm{O}$ ). ${ }^{1} \mathrm{H}$ NMR (200 MHz) 3.1-3.3 (m, 2H, CH$), 3.49-3.77\left(2 \mathrm{~s}, 3 \mathrm{H}, \mathrm{CH}_{3}\right), 3.82-3.91\left(\mathrm{~m}, 2 \mathrm{H}, \mathrm{CH}_{2}\right), 3.60-4.30$ $\left(\mathrm{m}, 3 \mathrm{H}, \mathrm{CH}_{2}+\mathrm{CH}\right), 5.00$ and $5.08(2 \mathrm{~d} J=4 J=3.3 \mathrm{~Hz}, 1 \mathrm{H}, \mathrm{CH}), 6.00(2 \mathrm{~d} J=4 J=3.3 \mathrm{~Hz}, 1 \mathrm{H}, \mathrm{CH})$, 7.01- $8.30(\mathrm{~m}, 9 \mathrm{H}, \mathrm{ArH}) \mathrm{ppm} .{ }^{13} \mathrm{C} \mathrm{NMR}(50 \mathrm{MHz}) 50.1\left(\mathrm{CH}_{2}\right), 52.8(\mathrm{CH}), 53.2\left(\mathrm{CH}_{2}\right), 64.7$ $\left(\mathrm{CH}_{3}\right), 78.9(\mathrm{CH}), 83.8(\mathrm{CH}), 115.6(\mathrm{CH}), 125.3(\mathrm{CH}), 127.1(\mathrm{CH}), 128.2(\mathrm{CH}), 129.9(\mathrm{CH})$, 136.5 (C), 144.8 (C), 157.1 (C), 171.7 (C) ppm. $\mathrm{C}_{19} \mathrm{H}_{19} \mathrm{~N}_{5} \mathrm{O}_{4} \mathrm{~S}$ (413.45): calcd. C 55.19, H 4.63, $\mathrm{N} 16.94 \%$ found C 55.02, H 4.80, N 16.74\%

\section{General procedure for the transformations dihydrotriazole-amidines}

Dihydrotriazole $4(5 \mathrm{mmol})$ was suspended in toluene $(10 \mathrm{~mL})$ and heated at reflux for $8 \mathrm{~h}$. At the end, the solution was evaporated under reduced pressure and the residue chromatographed with ethyl acetate-cyclohexane (1:4) yielding pure 5. 
Methyl 1-[1-(4-nitrophenylimino)-2-phenylethyl]pyrrolidine-2-carboxylate (5b). Yield 55\%. Light yellow oil. $[\alpha]_{\mathrm{D}}=+20.85 .{ }^{1} \mathrm{H}$ NMR $(200 \mathrm{MHz})$ 1.82-2.45 (m, 4H, 2CH$), 3.35-3.55(\mathrm{~m}, 2 \mathrm{H}$, $\left.\mathrm{CH}_{2} \mathrm{~N}\right), 3.55-3.82\left(\mathrm{~m}, 2 \mathrm{H}, \mathrm{CH}_{2} \mathrm{Ph}\right), 3.70\left(\mathrm{~s}, 3 \mathrm{H}, \mathrm{CH}_{3}\right), 4.50-4.68(\mathrm{~m}, 1 \mathrm{H}, \mathrm{CH}), 6.75(\mathrm{~d}, J=8.5 \mathrm{~Hz}$, $2 \mathrm{H}, \mathrm{ArH}), 7.15-7.39(\mathrm{~m}, 5 \mathrm{H}, \mathrm{ArH}), 8.04(\mathrm{~d}, J=8.5,2 \mathrm{H}, \mathrm{ArH}) \mathrm{ppm} .{ }^{13} \mathrm{C}$ NMR $(50 \mathrm{MHz}) 25.2$ $\left(\mathrm{CH}_{2}\right), 29.8\left(\mathrm{CH}_{2}\right), 35.6\left(\mathrm{CH}_{2}\right), 47.9\left(\mathrm{CH}_{2}\right), 52.4(\mathrm{CH}), 60.49\left(\mathrm{CH}_{3}\right), 122.8(\mathrm{CH}), 125.2(\mathrm{CH})$, $127.2(\mathrm{CH}), 128.2(\mathrm{CH}), 129.3(\mathrm{CH}), 135.4(\mathrm{C}), 142.7(\mathrm{C}), 155.4(\mathrm{C}), 158.1(\mathrm{C}), 174.0(\mathrm{C}) \mathrm{ppm}$. ESI-MS: $m / z 368$ [M+H]. $\mathrm{C}_{20} \mathrm{H}_{21} \mathrm{~N}_{3} \mathrm{O}_{4}$ (367.4): calcd. C 65.38, H 5.76, N 11.44\% found C 65.24, H 5.88, N $11.26 \%$

Methyl 3-[1-(4-nitrophenylimino)-2-phenylethyl]thiazolidine-4-carboxylate (5c). Yield 51\%. Light yellow oil. ${ }^{1} \mathrm{H}$ NMR (300 MHz) 3.15-3.36 (m, 2H, $\left.\mathrm{CH}_{2}\right), 3.60-3.91\left(\mathrm{~m}, 2 \mathrm{H}, \mathrm{CH}_{2} \mathrm{Ph}\right), 3.77$ $\left(\mathrm{s}, 3 \mathrm{H}, \mathrm{CH}_{3}\right), 4.41-4.48(\mathrm{~m}, 1 \mathrm{H}, \mathrm{CH}), 4.44-4.62(\mathrm{~m}, 1 \mathrm{H}, \mathrm{CH}), 5.00-5.21(\mathrm{~m}, 1 \mathrm{H}, \mathrm{CH}), 6.78(\mathrm{~d}$, $J=8.8,2 \mathrm{H}, \mathrm{ArH}), 7.17-7.37(\mathrm{~m}, 5 \mathrm{H}, \mathrm{ArH}), 8.06$ (d, $J=8.8,2 \mathrm{H}, \mathrm{ArH}) \mathrm{ppm} .{ }^{13} \mathrm{C}$ NMR $(75 \mathrm{MHz})$ $33.5\left(\mathrm{CH}_{2}\right), 35.7\left(\mathrm{CH}_{2}\right), 49.9\left(\mathrm{CH}_{2}\right), 52.8(\mathrm{CH}), 62.5\left(\mathrm{CH}_{3}\right), 122.3(\mathrm{CH}), 125.2(\mathrm{CH}), 127.4$ $(\mathrm{CH}), 128.0(\mathrm{CH}), 129.4(\mathrm{CH}), 134.6(\mathrm{C}), 143.0(\mathrm{C}), 154.8(\mathrm{C}), 156.9(\mathrm{C}), 171.3(\mathrm{C})$ ppm. ESIMS: $m / z 386[\mathrm{M}+\mathrm{H}] . \mathrm{C}_{19} \mathrm{H}_{19} \mathrm{~N}_{3} \mathrm{O}_{4} \mathrm{~S}$ (385.44): calcd. C 59.21, H 5.97, N 10.90\% found $\mathrm{C} 59.07$, H 5.03, N $10.88 \%$

General procedure for cyclization: Amidines $\mathbf{1 d - f , i}$ and $\mathbf{5 b , c}(1 \mathrm{mmol})$ were dissolved in $10 \mathrm{~mL}$ of anhydrous THF in $\mathrm{N}_{2}$ atmosphere. To the solution was added $1 \mathrm{~mL}$ of $t$-BuOK $1 \mathrm{M}$ solution. The stirred mixture was refluxed for 2-10 h until disappearance of the starting material [TLC ethyl acetate-cyclohexane (7:3) ]. The resulting solution was evaporated and the crude taken up with ethyl acetate and washed with aqueous acid. The neutral organic phase was dried on $\mathrm{Na}_{2} \mathrm{SO}_{4}$ and evaporated under reduced pressure. The residue was crystallized with the indicated solvent or, if necessary, chromatographed over a silica gel column (eluent from ethyl acetate-cycloexane 7:3 to ethyl acetate).

4-Methyl- $\boldsymbol{N}$-(5methyl-4-oxo-3-phenyl-4,5-dihydro-1 $\mathrm{H}$-pyrrol-2-yl)benzensolfonamide (6a). Yield 49\%. Mp $90{ }^{\circ} \mathrm{C}$ (light yellow crystals from $i$ - $\mathrm{Pr}_{2} \mathrm{O}$ ). ${ }^{1} \mathrm{H}$ NMR (200 MHz) 1.39 (d $J=7.9$, $\left.3 \mathrm{H}, \mathrm{CH}_{3}\right), 2.41\left(\mathrm{~s}, 3 \mathrm{H}, \mathrm{CH}_{3} \mathrm{Ph}\right), 2.75-4.10$ (bs, 2H, 2NH), 4.00-4.21 (m, 1H, CH), 7.16-7.83 (m, 9H, ArH) ppm. ${ }^{13} \mathrm{C}$ NMR (50 MHz) $17.6\left(\mathrm{CH}_{3}\right), 21.7\left(\mathrm{CH}_{3}\right), 57.1(\mathrm{CH}), 104.8(\mathrm{C}), 126.6(\mathrm{CH})$, $127.2(\mathrm{CH}), 128.6(\mathrm{CH}), 128.9(\mathrm{CH}), 129.6(\mathrm{CH}), 129.9(\mathrm{C}), 139.0(\mathrm{C}), 143.5(\mathrm{C}), 166.1(\mathrm{C})$, 180.4 (C) ppm. ESI-MS: $m / z 343[\mathrm{M}+\mathrm{H}] . \quad \mathrm{C}_{18} \mathrm{H}_{18} \mathrm{~N}_{2} \mathrm{O}_{3} \mathrm{~S}$ (342.41): calcd. C 63.14, H 5.30, N $8.18 \%$ found $\mathrm{C} 62.98, \mathrm{H} 5,45, \mathrm{~N} 8.06 \%$

$\mathrm{N}$-(5-Isopropyl-4-oxo-3-phenyl-4,5-dihydro-1 $\mathrm{H}$-pyrrol-2-yl)-4-methylbenzensulfonamide (6b). Yield 58\%. Glassy solid. ${ }^{1} \mathrm{H}$ NMR $(200 \mathrm{MHz}) 0.65\left(\mathrm{~d} J=6.6 \mathrm{~Hz}, 3 \mathrm{H}, \mathrm{CH}_{3}\right), 1.02$ (d $J=7.0$, $\left.3 \mathrm{H}, \mathrm{CH}_{3}\right), 2.03-2.36(\mathrm{~m}, 1 \mathrm{H}, \mathrm{CH}), 2.40$ (s, 3H, $\left.\mathrm{CH}_{3} \mathrm{Ph}\right), 2.60-3.45$ (bs, 2H, 2NH), 3.95-4.02 (m, 1H, CH), 7.21-7.89 (m, 9H, ArH) ppm . ${ }^{13} \mathrm{C}$ NMR (50 MHz) 14.7 $\left(\mathrm{CH}_{3}\right), 20.7\left(\mathrm{CH}_{3}\right), 29.9(\mathrm{CH})$, $66.1(\mathrm{CH}), 106.8(\mathrm{C}), 126.7(\mathrm{CH}), 127.3(\mathrm{CH}), 128.7(\mathrm{CH}), 128.9(\mathrm{CH}), 129.7(\mathrm{CH}), 135.6(\mathrm{C})$, 138.8 (C), 142.6 (C), 167.1 (C), 203.0 (C) ppm. ESI-MS: $m / z 371$ [M+Na], 369 [M-H]. $\mathrm{C}_{20} \mathrm{H}_{22} \mathrm{~N}_{2} \mathrm{O}_{3} \mathrm{~S}$ (370.43): calcd. C 64.84, H 5.99, N 7.56\% found C 64.75, H 6.08, N 7.34\% 
4-Methyl- $\boldsymbol{N}$-(4-oxo-3-phenyl-4,5-dihydro-1H-pyrrol-2-yl)benzensulfonamide (6c). Yield $82 \%$. Mp $85{ }^{\circ} \mathrm{C}$ (light yellow crystal from $i$ - $\mathrm{Pr}_{2} \mathrm{O}$ ). ${ }^{1} \mathrm{H}$ NMR (300 MHz) 2.38 (s, 3H, CH3), 3.004.40 (bs, 2H, 2NH), 3.90-4.10 (m, 2H, CH2), 7.07-7.81 (m, 9H, ArH) ppm. ${ }^{13} \mathrm{C} \mathrm{NMR} \mathrm{(75} \mathrm{MHz)}$ $21.6\left(\mathrm{CH}_{3}\right), 50.5\left(\mathrm{CH}_{2}\right), 106.7(\mathrm{C}), 126.4(\mathrm{CH}), 127.0(\mathrm{CH}), 128.2(\mathrm{CH}), 128.8(\mathrm{CH}), 129.7$ (CH), 130.1 (C), 139.5 (C), 143.2 (C), 168.8 (C), 174.3 (C) ppm. ESI-MS: m/z 327 [M-H]. $\mathrm{C}_{17} \mathrm{H}_{16} \mathrm{~N}_{2} \mathrm{O}_{3} \mathrm{~S}$ (328.39): calcd. C 62.18, H 4.91, N 8.53\% found C 62.05, H 5.11, N 8.39\%

$\mathrm{N}$-[5-(2-Mercaptoethyl)-4-oxo-3-phenyl-4,5-dihydro-1 $\mathrm{H}$-pyrrol-2-yl]-4-methylbenzen sulfonamide (6d). Yield 36\%. Mp 98-100 ${ }^{\circ} \mathrm{C}$ (light yellow crystal from $\left.i \operatorname{Pr}_{2} \mathrm{O}\right) .{ }^{1} \mathrm{H}$ NMR (200 $\mathrm{MHz}) 2.41$ (s, 3H, $\left.\mathrm{CH}_{3} \mathrm{Ph}\right), 2.60-3.45$ (bs, 2H, 2NH), 2.87-3.17 (m, 4H, 2CH$)$, 4.28-4.39 (m, 1H, CH), 7.11-8.17 (m, 9H, ArH), 8.11-8.17 (bs, 1H, SH) ppm. ${ }^{13} \mathrm{C}$ NMR (50 MHz) $21.7\left(\mathrm{CH}_{3}\right)$, $31.6\left(\mathrm{CH}_{2}\right), 34.0\left(\mathrm{CH}_{2}\right), 60.9(\mathrm{CH}), 101.6(\mathrm{C}), 126.5(\mathrm{CH}), 127.8(\mathrm{CH}), 128.9(\mathrm{CH}), 129.6(\mathrm{CH})$, 130.8 (CH), 134.3 (C), 134.1 (C), 139.3 (C), 167.3 (C), 170.0 (C) ppm. ESI-MS: m/z 389 $[\mathrm{M}+\mathrm{H}] . \quad \mathrm{C}_{19} \mathrm{H}_{20} \mathrm{~N}_{2} \mathrm{O}_{3} \mathrm{~S}_{2}$ (388.5): calcd. C 58.74, $\mathrm{H} \mathrm{5.19,} \mathrm{N} 7.21 \%$ found $\mathrm{C} 58.60, \mathrm{H} \mathrm{5.33,} \mathrm{N}$ $7.15 \%$

3-(4-Nitrophenylamino)-2-phenyl-5,6,7,7a-tetrahydropyrriolizin-1-one (7a). Yield 45\%. Mp $235^{\circ} \mathrm{C}$ (light yellow crystals from $i$ - $\mathrm{Pr}_{2} \mathrm{O}$ ). ${ }^{1} \mathrm{H}$ NMR (DMSO, $\left.300 \mathrm{MHz}\right) 1.75-1.81(\mathrm{~m}, 1 \mathrm{H}, \mathrm{CH})$, 1.82-2.05 (m, 2H, $\left.\mathrm{CH}_{2}\right), 2.12-2.36(\mathrm{~m}, 1 \mathrm{H}, \mathrm{CH}), 2.81-3.12\left(\mathrm{~m}, 2 \mathrm{H}, \mathrm{CH}_{2}\right), 4.08-4.22(\mathrm{~m}, 1 \mathrm{H}$, $\mathrm{CHN}$ ), 7.02-8.18 (m, 9H, ArH), 10.18 (bs, 1H, NH) ppm. ${ }^{13} \mathrm{C}$ NMR (DMSO, $\left.75 \mathrm{MHz}\right) 27.4$ $\left(\mathrm{CH}_{2}\right), 27.5\left(\mathrm{CH}_{2}\right), 49.4\left(\mathrm{CH}_{2}\right), 70.5(\mathrm{CH}), 102.6(\mathrm{C}), 119.6(\mathrm{CH}), 125.9(\mathrm{CH}), 127.9(\mathrm{CH}), 128.7$ $(\mathrm{CH}), 130.1(\mathrm{CH}), 133(\mathrm{C}), 141.9(\mathrm{C}), 147.4(\mathrm{C}), 169.4(\mathrm{C}), 197.1$ (C) ppm. ESI-MS: m/z 335 $[\mathrm{M}+\mathrm{H}] . \mathrm{C}_{19} \mathrm{H}_{17} \mathrm{~N}_{3} \mathrm{O}_{3}$ (335.36): calcd. C 68.05, H 5.11, N 12.53\% found $\mathrm{C} 67.89, \mathrm{H} \mathrm{5.26,} \mathrm{N}$ $12.48 \%$

4-(4-Nitrophenylkamino)-6-phenyl-1,7-dihydropyrrolo[1,2-c]thiazol-7(3H)-one (7b). yield $39 \%$. Mp $110{ }^{\circ} \mathrm{C}$ (light yellow crystals from $i$-Pr $2 \mathrm{O}$ ). ${ }^{1} \mathrm{H}$ NMR (DMSO, $200 \mathrm{MHz}$ ) 3.06-3.38 (m, $\left.2 \mathrm{H}, \mathrm{CH}_{2}\right), 3.88-4.61\left(\mathrm{~m}, 3 \mathrm{H}, \mathrm{CH}\right.$ and $\left.\mathrm{CH}_{2}\right), 6.94-8.05(\mathrm{~m}, 9 \mathrm{H}, \mathrm{ArH}), 10.34(\mathrm{bs}, 1 \mathrm{H}, \mathrm{NH}) \mathrm{ppm}$. ${ }^{13} \mathrm{C}$ NMR (DMSO, $\left.50 \mathrm{MHz}\right) 32.2\left(\mathrm{CH}_{2}\right), 53.8\left(\mathrm{CH}_{2}\right), 70.5(\mathrm{CH}), 106.0(\mathrm{C}), 119.8(\mathrm{CH}), 125.7$ $(\mathrm{CH}), 126.5(\mathrm{CH}), 128.0(\mathrm{CH}), 128.7(\mathrm{CH}), 132.0(\mathrm{C}), 142.1(\mathrm{C}), 147.0(\mathrm{C}), 168.6(\mathrm{C}), 196.9(\mathrm{C})$ ppm. ESI-MS: $m / z 354[\mathrm{M}+\mathrm{H}] . \mathrm{C}_{18} \mathrm{H}_{15} \mathrm{~N}_{3} \mathrm{O}_{3} \mathrm{~S}$ (353.39): calcd. C 61.02, $\mathrm{H} 4.33$, N 11.69\% found C $60.88, \mathrm{H} 4.33$, N $11.69 \%$

\section{Acknowledgements}

We thank Ministero dell'Istruzione e della Ricerca Universitaria (MIUR) for financial support.

\section{References}

1. Cassani, F.; Celentano, G.; Erba, E.; Pocar, D. Synthesis 2004, 1041.

2. (a) Clerici, F.; Gelmi, M. L.; Rossi, L. M. Synthesis 1987, 1025. (b) Battistini, M.; Erba, E.; Pocar, D. Synthesis, 1992, 1206. (c) Pocar, D.; Roversi, E.; Trimarco, P.; 
Valgattarri,G. Liebigs Ann. 1995, 487. (d) Erba, E.; Pocar, D.; Trimarco, P. J. Chem. Soc., Perkin Trans. 1 1998, 3535.

3. Fusco, R.; Bianchetti, G.; Pocar, D.; Ugo, R. Chem. Ber. 1963, 96, 802.

4. Beccalli, E. M.; Erba, E.; Gelmi, M. L.; Pocar, D. J. Chem. Soc., Perkin Trans. 1 1996, 1359.

5. Friedrichsen, W.; Traulsen, T.; Elguero, J.; Katritzky, A. R. Adv. Heterocycl. Chem. 2006, 76, 115. 